\title{
Cancer stem cells in the development of liver cancer
}

\author{
Taro Yamashita ${ }^{1}$ and Xin Wei Wang ${ }^{2}$ \\ 1Department of General Medicine, Kanazawa University Hospital, Kanazawa, Ishikawa, Japan. \\ ${ }^{2}$ Laboratory of Human Carcinogenesis, Center for Cancer Research, National Cancer Institute, Bethesda, Maryland, USA.
}

\begin{abstract}
Liver cancer is an aggressive disease with a poor outcome. Several hepatic stem/progenitor markers are useful for isolating a subset of liver cells with stem cell features, known as cancer stem cells (CSCs). These cells are responsible for tumor relapse, metastasis, and chemoresistance. Liver CSCs dictate a hierarchical organization that is shared in both organogenesis and tumorigenesis. An increased understanding of the molecular signaling events that regulate cellular hierarchy and stemness, and success in defining key CSC-specific genes, have opened up new avenues to accelerate the development of novel diagnostic and treatment strategies. This Review highlights recent advances in understanding the pathogenesis of liver CSCs and discusses unanswered questions about the concept of liver CSCs.
\end{abstract}

\section{The cancer stem cell concept}

Although considered monoclonal in origin, tumor cells show heterogeneous morphology and behavior $(1,2)$. This heterogeneity has traditionally been explained by the clonal evolution of tumor cells resulting from the progressive accumulation of multiple genetic (3) or epigenetic changes (4). Alterations in tumor stroma microenvironments may also facilitate the development of tumor cell heterogeneity through the extrinsic activation of certain tumor cell signaling pathways (5). Moreover, recent studies have suggested that heterogeneity is a result of the hierarchical organization of tumor cells by a subset of cells with stem/progenitor cell features known as cancer stem cells (CSCs) (6).

The concept of cancer as an abnormal stem cell disease was proposed based on the similar capabilities of cancer cells and normal stem cells to self-renew, produce heterogeneous progeny, and divide in an unlimited fashion $(7,8)$. However, the CSC hypothesis has only recently been experimentally validated by the identification of a subset of certain self-renewing stem cell marker-positive cells with a hierarchical organization (9-11). The self-renewal capacity is confirmed by serial in vitro clonogenic growth and in vivo tumorigenicity; thus, CSCs are also known as tumor-initiating cells or tumor-propagating cells. CSCs are highly tumorigenic, metastatic, chemotherapy and radiation resistant, responsible for tumor relapse after therapy, and able to divide symmetrically and asymmetrically to orchestrate the tumor mass (11). Therefore, CSCs are a pivotal target for the eradication of many cancers including liver cancer.

Liver cancer is the fifth most commonly diagnosed cancer and the second most frequent cause of cancer death in men worldwide (12). Among primary liver cancers, hepatocellular carcinoma (HCC) represents the major histological subtype, accounting for $70 \%-85 \%$ of cases of primary liver cancer (12). Intrahepatic cholangiocarcinoma (ICC) is the second most frequent type of liver cancer, and its incidence has been increasing $(12,13)$. Both HCC and ICC are heterogeneous diseases in terms of cellular morphology and clinical outcome. Combined HCC-cholangiocellular carcinoma (HCC-CCA), a form of primary liver cancer showing features of both hepatocellular and biliary epithelial differentiation,

Conflict of interest: The authors have declared that no conflict of interest exists. Citation for this article: J Clin Invest. 2013;123(5):1911-1918. doi:10.1172/JCI66024. has also been reported, supporting the existence of bipotent liver CSCs (14). Indeed, recent immunohistochemical studies of stem cell markers suggest that HCC, ICC, and HCC-CCA are histologically heterogeneous and contain a subset of cells expressing a variety of stem cell markers (15-18).

CSC self-renewal and hierarchical organization features have been experimentally validated by xenotransplantation of freshly resected HCC specimens. In HCC, CSC markers include epithelial cell adhesion molecule (EpCAM), CD133, CD90, CD44, CD24, CD13, and oval cell marker OV6, as well as Hoechst dye efflux or aldehyde dehydrogenase activities, some of which may functionally support liver CSC phenotypes including highly invasive features and chemoresistance (18-24). This Review summarizes the current knowledge of liver CSCs and discusses several unanswered questions about the concept of liver CSCs.

\section{Liver microenvironment and the CSC niche}

Liver cancer nearly always develops in the setting of chronic liver disease (CLD), in which continuous inflammation and hepatocyte regeneration occur (25). Pathophysiological changes take place during long-term inflammation/regeneration processes that work coordinately to initiate and/or promote liver cancer. These processes include the expansion of stem/progenitor cells, accumulation of genetic and/or epigenetic changes, and alteration of the microenvironment (Figure 1).

Hepatic stem/progenitor cells are markedly elevated in CLDs (26). Under selected circumstances, the hepatocyte proliferative capacity is considered virtually infinite (27). However, in human CLDs, this capacity is impaired, possibly due to hepatitis virus infection (28) or replicative senescence induced by long-term continuous hepatocyte regeneration (29). This impairment in hepatocyte proliferation may cause the expansion of stem/progenitor cells called "ductular reactions" (30). Hepatic stem/progenitor cells derive from the canals of Hering, bile canaliculi lined with hepatocytes and cholangiocytes (31). Stem cell homing, motility, and proliferation are tightly regulated by the immediate microenvironment termed the stem cell niche (32). In the liver, the niche cells that control self-renewal and division of hepatic stem/progenitors have not yet been clarified. The magnitude of progenitor cell activation seems to correspond to the severity of liver fibrosis and inflammation (30) and correlate with HCC risk (33). 


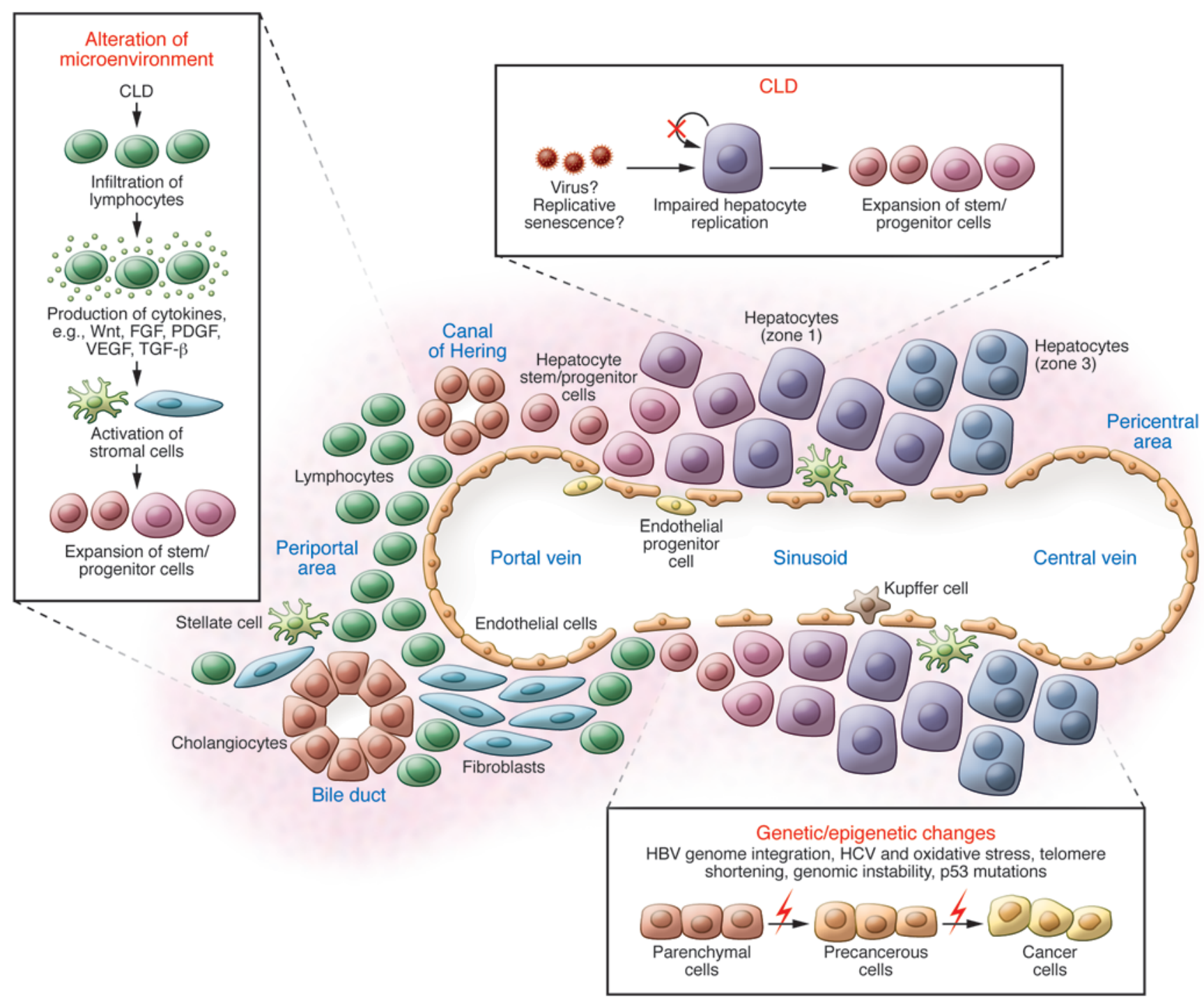

\section{Figure 1}

Liver inflammation and regeneration in liver CSC development. Stem/progenitor cells expand in CLD as a result of impaired hepatocyte replication, and genetic and epigenetic changes potentially accumulate in all liver lineages. Activation of stromal cells may induce various signaling pathways, including cytokines such as Wnt, FGF, PDGF, VEGF, and TGF- $\beta$, and promote the development of liver CSCs.

Genetic and epigenetic changes accumulate in all liver lineages over decades and are responsible for initiating and promoting liver cancer. HBV infection results in HBV genome integration into the host genome and may initiate and promote $\mathrm{HCC}$ by inducing insertional mutagenesis and genomic instability (34). The HBV X gene (HBx) also modulates signaling pathways, including p 53 and NF- $\kappa \mathrm{B}$, to promote HCC (35). HCV infection may enhance the induction of ROS by modulating mitochondrial functions, resulting in DNA damage (36). Telomere shortening as a consequence of accelerated hepatocyte turnover also contributes to genomic instability and HCC (37). TP53 mutations, especially codon $249^{\mathrm{Ser}}$ mutations following aflatoxin B1 exposure, result in defective DNA damage responses (38). These oncogenic events may occur simultaneously in various populations of hepatic stem/progenitor cells and hepatocytes in CLDs.

The hepatic microenvironment is drastically altered in CLDs, with increased lymphocyte infiltration, stellate cell activation, and the expansion of hepatic progenitor cells and endothelial progenitor cells. Infiltrating lymphocytes cause inflammation with the release of free radicals, cytokines, and chemokines, resulting in DNA damage, cell proliferation, and migration $(38,39)$. Activation of stellate cells by TGF- $\beta$, possibly secreted from infiltrating lymphocytes, Kupffer cells, or damaged hepatocytes, results in fibrosis with excess deposition of extracellular matrix (40). Activated myofibroblasts in turn produce growth factors such as Wnt, FGF, and PDGF to regulate cell proliferation (41, 42). Endothelial progenitor cells and sinusoidal endothelial cells may migrate, proliferate, and subsequently mediate vasculature reconstruction during liver regeneration in part through interaction of VEGF and its receptor VEGFR $(43,44)$.

Taken together, stromal cell activation appears to induce signaling pathways in a range of liver lineages that emerge in CLDs with accumulating genetic and/or epigenetic changes. These patho- 


\section{Table 1}

Signaling pathways activated in hepatic stem/progenitor cells

\begin{tabular}{|c|c|c|c|}
\hline Signaling pathway & Role in normal liver development (species) & Role in liver cancer development (source) & Reference \\
\hline BMP & Liver specification from endoderm (mouse) & $\begin{array}{l}\text { Differentiation of CD133+ CSC } \\
\text { (PLC/PRL/5, Huh7, MHCC-97L) }\end{array}$ & 46,66 \\
\hline FGF & Liver specification from endoderm (mouse) & $\begin{array}{l}\text { Cell proliferation and angiogenesis } \\
\text { (HCC-1.2, HepG2, Hep3B) }\end{array}$ & $45,53,54$ \\
\hline Wnt & $\begin{array}{c}\text { Liver specification from endoderm (zebrafish, mouse), } \\
\text { inhibition of hepatic maturation (mouse), } \\
\text { biliary differentiation of hepatoblast (mouse) }\end{array}$ & $\begin{array}{l}\text { Liver CSC self-renewal (Huh1, Huh7, } \\
\text { SMMC7721), cell proliferation (Hep3B) }\end{array}$ & $\begin{array}{c}18,47,48,51 \\
54,58,59\end{array}$ \\
\hline OSM & Hepatocytic differentiation of hepatoblast (mouse) & $\begin{array}{l}\text { Differentiation of EpCAM+ CSC } \\
\text { (Huh1, Huh7, primary HCC) }\end{array}$ & 49,67 \\
\hline TGF- $\beta$ & Biliary differentiation of hepatoblast (mouse) & Controversial (mouse, rat, Huh7, primary HCC) & $64,65,68-73$ \\
\hline JAG1/Notch & Biliary differentiation of hepatoblast (mouse) & Controversial (mouse, primary HCC) & $50,78,79$ \\
\hline IL-6/STAT3 & Liver regeneration (mouse) & Liver CSC maintenance (mouse) & $70,76,77$ \\
\hline HGF/c-Met & $\begin{array}{l}\text { Liver regeneration (mouse), hepatocyte } \\
\text { transdifferentiation into biliary epithelium (rat) }\end{array}$ & Epithelial-mesenchymal transition (mouse) & $49,50,80-82$ \\
\hline
\end{tabular}

physiological conditions may cooperatively affect the process of liver regeneration and may support liver CSC development.

\section{Shared features of liver development and liver cancer development}

Embryogenesis and tumorigenesis share similar features, including autonomous cell proliferation, motility, homing, dynamic morphologic changes, cellular heterogeneity, and interactions with the microenvironment. Indeed, carcinogenesis could be described as deregulated malignant organogenesis mediated by abnormally proliferating and/or metastatic cancer cells and activated stromal cells that trigger angiogenesis, fibrosis, and inflammation. Liver cancer development recapitulates, in part, fetal liver development in terms of the emergence of cells expressing certain stem cell markers and the activation of signaling pathways during liver development and inflammation/regeneration (Table 1).

Normal liver development program. Hepatic genes are first induced in a segment of ventral endoderm, requiring FGF signaling from the adjacent cardiogenic mesoderm and BMP signaling from the septum transversum $(45,46)$. Recent analysis of zebrafish indicates the involvement of Wnt $2 \mathrm{~b}$ from the lateral plate mesoderm in liver specification (47). Another recent study using conditional adenomatosis polyposis coli $(A p c)$ knockout mice under the $\alpha$-fetoprotein $(A f p)$ promoter further indicated that activation of Wnt signaling allows the induction of hepatic specification and inhibition of hepatic maturation (48). Once the hepatic endoderm is specified and the liver bud begins to grow, the cells become hepatoblasts with the ability to differentiate into hepatic and biliary lineages. These cells have the self-renewal and asymmetric division features of stems cells and can repopulate normal and injured liver. Various growth factors influence hepatoblast differentiation into hepatocytes and cholangiocytes, including Wnt signaling, HGF signaling, oncostatin M (OSM) signaling, and jagged 1/ Notch (JAG1/Notch) signaling (49-51).

Signaling pathways activated in liver cancer. Signaling pathways activated in normal liver development are also activated in CLDs and may mediate the development and maintenance of liver CSCs (Table 2) (see also a recent review article highlighting the role of signaling pathways on self-renewal and differentiation of liver
CSCs; ref. 52). For example, signaling of FGF and Wnt is implicated in HCC development (53-55), and Wnt signaling regulates hepatoblasts and liver CSC self-renewal $(18,56-60)$. Recent application of massive parallel sequencing technologies have consistently confirmed previous findings that somatic mutations of genes in the $\mathrm{Wnt} / \beta$-catenin signaling pathways, such as axin and $\beta$-catenin, are common HCC events (61-63). Wnt and TGF- $\beta$ signaling pathways may collaborate in the development of HCC or HCC-CCA with poor prognosis $(64,65)$. Signaling of OSM and BMP4 appears to induce hepatocytic differentiation of liver CSCs $(66,67)$, while TGF- $\beta$ signaling is implicated in the biliary differentiation of hepatoblasts; loss of TGF- $\beta$ signaling by $\beta 2$-spectrin knockout resulted in the expansion of progenitor cells in mice (68, $69)$. TGF- $\beta$ signaling may also regulate the development and maintenance of HCC and liver CSCs, but its role seems paradoxical and is often referred to as a double-edged sword (70-73). In addition, TGF- $\beta$ signaling may promote HCC progression by recruiting regulatory $\mathrm{T}$ cells to establish a favorable microenvironment for tumor metastasis $(74,75)$.

IL-6/STAT3 signaling is involved in liver inflammation/regeneration and may regulate the population of liver CSCs in collaboration with TGF- $\beta$ signaling $(70,76,77)$. JAG1/Notch signaling induces biliary differentiation of hepatoblasts, but its role in HCC remains controversial $(78,79)$. Signaling through HGF and its receptor c-Met has a pleiotropic role in regulating hepatic progenitors to hepatocytic/biliary differentiation $(80,81)$ and is implicated in the maintenance of liver CSCs through epithelialmesenchymal transition (82).

Stem cell markers detected in hepatic stem/progenitor cells and liver CSCs. Hepatoblasts express biliary markers such as cytokeratin 19 (CK19) and EpCAM as well as hepatocyte markers such as albumin and $\operatorname{AFP}(27,83)$. Hepatoblasts also express a variety of markers putatively detected in ectodermal or mesodermal lineages (84-92). Similar to the signaling pathways activated in both normal liver and cancer development, hepatoblasts/hepatic progenitor cells, and liver CSCs share a number of oncofetal markers (Tables 1 and 2 and refs. 17, 18, 20, 21, 27, 58, 83, 93-98). Interestingly, recent studies showed that some of these liver CSC markers are also functionally involved in the maintenance of 
Table 2

Cell surface markers activated in liver CSCs

\begin{tabular}{lr} 
Cell surface marker & \multicolumn{1}{c}{ Function in CSCs } \\
CD13 & ROS-induced DNA damage reduction \\
CD133 & Neurotensin/IL-8/CXCL1 signaling \\
CD24 & STAT3-mediated NANOG regulation \\
CD44 & Regulation of redox status through XCT \\
CD90 & Not reported \\
DLK1 & Not reported \\
EpCAM & Activation of Wnt signaling \\
OV6 & Not reported
\end{tabular}

\author{
Phenotypes of marker-positive CSCs (source) \\ Tumorigenic, cell cycle arrest, chemoresistant \\ (PLC/PRL/5, Huh7, Hep3B) \\ Tumorigenic, chemoresistant \\ (PLC8024, Huh7, Hep3B, primary HCC) \\ Tumorigenic, chemoresistant, metastatic \\ (PLC/PRL/5, HLE, Huh7, primary HCC) \\ Tumorigenic, invasive (PLC, PLC/PRL/5, HLF) \\ Tumorigenic, metastatic, circulating (HepG2, Hep3B, \\ PLC, Huh7, MHCC97L, MHCC97H, primary HCC) \\ Tumorigenic, chemoresistant (Hep3B, Huh7) \\ Tumorigenic, invasive, chemoresistant, circulating \\ (Huh1, Huh7, primary HCC) \\ Tumorigenic, chemoresistant, invasive, metastatic
}

(Huh7, SMMC7721, primary HCC)
Reference

19

$17,88,101$

$128,130,131$

21,96

102,122

$20,116,125$

98

$18,56,57,60$,

$99,100,127$

23,58
CSC features. For example, EpCAM enhances Wnt signaling in ES cells and cancer $(99,100)$, and CD133 expression is required for the maintenance of CD $133^{+}$liver CSCs through neurotensin/ IL-8/CXCL1 signaling activation (101). In addition, a CD44 variant regulates the redox status by stabilizing $\mathrm{xCT}$ and protecting CSCs from oxidative stress (102), while CD13 reduces cell damage induced by oxidative stress after exposure to genotoxic reagents (19). Thus, the functional involvement of most liver CSC markers in the maintenance of liver CSC features potentially makes them a good target for the eradication of liver CSCs.

Reprogramming factors and liver cancer development. As Yamanaka and colleagues elegantly demonstrated, differentiated cells can be reprogrammed to acquire pluripotency by defined factors such as Oct3/4, Sox2, Klf4, and c-Myc (the Yamanaka factors) known to be activated in ES cells $(103,104)$. These transcription regulators may also play a role in HCC and liver CSC development and maintenance. Recently, liver CSCs were observed in Myc-driven but not Akt/Ras-driven tumors, suggesting a role for Myc in liver CSC development and maintenance (105). Nanog, a transcription regulator activated in ES cells, plays a role in the self-renewal of liver CSCs expressing CD24 or CD133 $(21,106)$, while Sox2, Oct4, and Nanog expression may predict the prognosis of HCC patients after surgery, suggesting roles in HCC malignant phenotypes $(107,108)$. TP53 mutations are the most common genetic alteration associated with stem cell-like gene expression signatures in HCC (109), and loss of p53 function enhances the efficiency of Yamanaka factorinduced reprogramming (110-112). These data together suggest that factors affecting cellular reprogramming and inducing dedifferentiation may also work as oncogenes to generate liver CSCs.

Heterogeneity of normal hepatic stem/progenitor cells and liver CSCs. Hepatoblasts are considered a heterogeneous population with potential hierarchical organization and various degrees of differentiation (113). Therefore, many controversial issues including differentiation status and the repopulation capability of markerpositive hepatoblasts have been raised, even using robust stem cell markers $(95,114,115)$. Similarly, liver CSCs are considered highly heterogeneous and may show distinct phenotypes in terms of tumorigenic/metastatic features and chemosensitivity when purified using different CSC markers (116). Therefore, the expression patterns and levels of these CSC markers in primary liver cancers need to be comprehensively clarified to aid the development of molecularly targeted therapies against liver CSCs.

\section{Clinical application of the concept of liver CSCs}

According to the CSC concept, heterogeneous populations of liver cancer cells are dictated and maintained at least partially by liver CSCs. Therefore, identification of signaling pathways as well as stem cell markers activated in liver CSCs will profoundly affect the development of novel liver cancer diagnosis and treatment strategies.

Diagnosis and prognostic stratification. Survival of liver cancer patients after radical resection varies on an individual basis, even with early diagnosis. Conventional histologic diagnosis of tumor samples cannot predict the prognosis of liver cancer patients who receive radical treatment. To overcome these limitations, gene expression profiling technologies have been applied $(117,118)$. Currently, the finding of a stem cell-like gene expression signature is of great interest because it reflects the malignant nature of a tumor with poor survival outcome (Figure 2 and refs. 18, 109, $119,120)$. Gene expression profiles generally reflect the characteristics of the dominant cell population, so a poor prognosis of HCC in patients with stemness-associated gene expression traits is assumed to reflect the abundance of liver CSCs with highly tumorigenic and/or metastatic features.

Accordingly, recent evidence has suggested that the presence of liver CSCs in resected specimens could be associated with poor prognosis of HCC patients after radical resection $(121,122)$. However, predictive values of single liver CSC markers remain controversial and need validation in independent cohorts (52). Rather, a combination of several CSC markers may provide greater specificity and reliability in predicting HCC prognosis $(57,123)$. Stemness has also recently been identified as a predictive marker of ICC prognosis (124). CSCs have a highly invasive and metastatic capacity and can be isolated from peripheral blood mononuclear cells as circulating tumor cells, and thus may provide diagnostic or prognostic information (125-127).

Treatment resistance and CSC-targeted therapy. The discovery of liver CSCs has also elucidated detailed mechanisms of treatment failure in liver cancer. Poor prognosis after radical resection of $\mathrm{EPCAM}^{+} \mathrm{AFP}^{+}$ 
Hepatobiliary lineage commitment

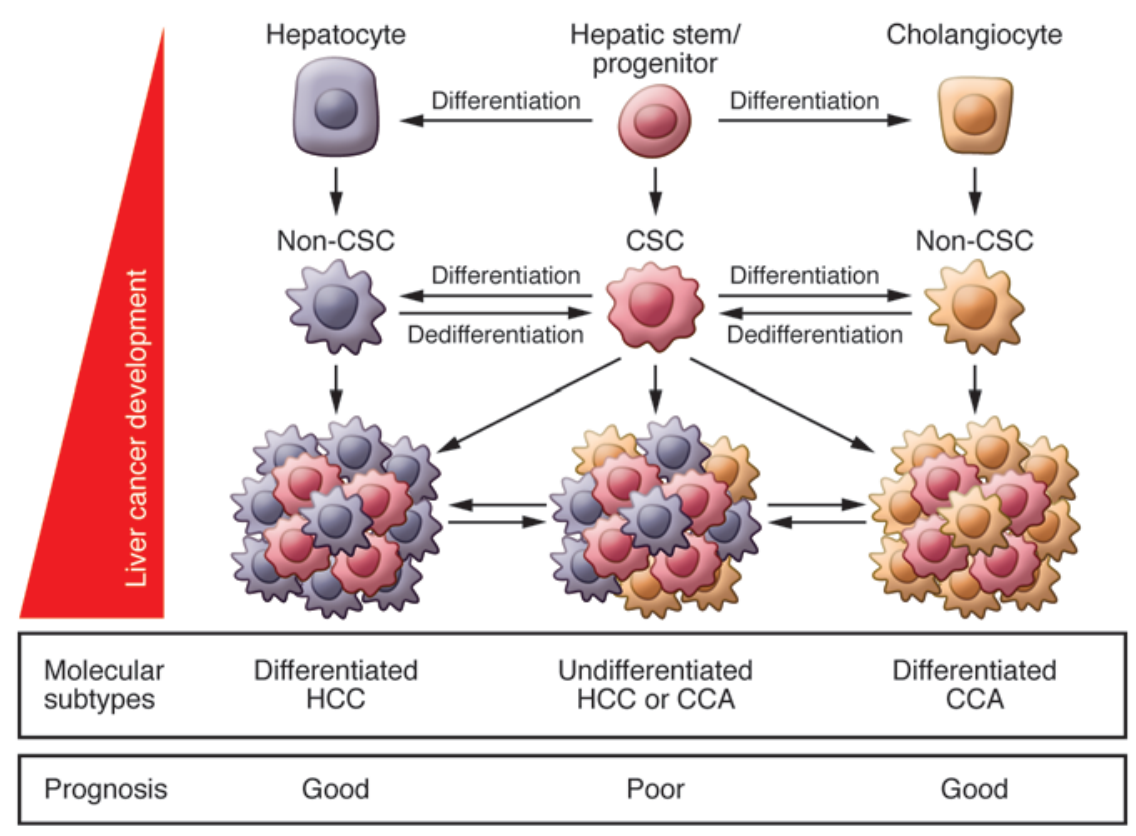

Figure 2

Potential origin and evolution of liver CSCs. CSCs may originate from non-CSCs by the activation of dedifferentiation programs. Liver CSC development may be regulated by hepatobiliary lineage commitment programs and oncogenic programs that are induced by acquired by genetic/epigenetic changes and activated signaling pathways. The emergence and domination of liver CSCs may reflect the molecular subtypes of liver cancers linked to the clinical outcome. CCA, cholangiocellular carcinoma.

HCCs can be explained by the high frequency of portal vein invasion, which may result in the early recurrence of HCC due to microdissemination in the residual liver $(18,57)$. Although EpCAM ${ }^{+}$CSCs showed chemoresistance against genotoxic reagent 5-fluorouracil (5-FU), these cells exhibited Wnt signaling activation and sensitivity to Wnt signaling inhibitor (56). CD90 ${ }^{+}$liver CSCs co-expressing CD44 were detected in all HCC tissues from 13 HCC patients who underwent surgery (20). These cells lost their highly tumorigenic capacity in a xenotransplantation model when anti-CD44-neutralizing antibodies were systematically administrated (20).

Transcatheter arterial chemoembolization (TACE) is used to treat HCC patients at intermediate stages, and CD $13^{+}$liver CSCs survived together with the fibrous capsule after TACE, which may result in local recurrence (19). Such $\mathrm{CD} 13^{+}$CSCs were eradicated by application of a CD13 inhibitor in combination with 5-FU in a mouse xenograft model (19). Among HBV-related HCC patients who received surgery, $\mathrm{CD} 133^{+}$liver CSCs appeared in advanced stages and correlated with early recurrence and poor prognosis (128). In advanced stages, genotoxic agents such as 5-FU or doxorubicin showed limited antitumor activities against HCC, and sorafenib tosylate only modestly prolonged overall survival (129). These $\mathrm{CD}_{133}{ }^{+}$liver CSCs showed activation of the Akt/PKB survival pathway and chemoresistance to doxorubicin or 5-FU, while an AKT1 inhibitor suppressed survival signaling of CD $133^{+}$liver CSCs (130). Lupeol, a phytochemical in fruit and vegetables, also inhibits the self-renewal of CD $133^{+}$liver CSCs through inhibition of the PTEN/ Akt/ABCG2 signaling pathway (131).
The discovery and exploration of liver CSCs has expanded our knowledge of the mechanisms by which liver cancers obtain tumorigenic, metastatic, and chemotherapy- and radiation-resistant capacities. The development of new diagnostic and treatment strategies targeting liver CSCs to improve the survival of liver cancer patients is currently underway.

\section{Future challenges}

The liver CSC concept has been acknowledged to explain the molecular diversity of malignant phenotypes in liver cancer. However, many questions remain, including the role of hepatitis viruses, the origin of liver CSCs, the existence and role of the stem cell niche, similarities and differences between normal hepatic stem/progenitor cells and liver CSCs, the timing of CSC emergence, CSC concept universality in liver cancers, and the relationship between CSC plasticity and clonal evolution accompanied by genetic and epigenetic changes (11).

Although chronic HBV and HCV infections are two major risk factors for the development of liver cancer, their roles in liver CSCs are largely unknown. It is also unclear whether HBV and HCV infect and replicate in hepatic stem/progenitor cells, but a recent study suggested that $\mathrm{HCV}$ could replicate in human fetal hepatocytes (132). Clinicopathological analysis of surgically resected $\mathrm{HCC}$ specimens suggested that $\mathrm{EPCAM}^{+} \mathrm{CSCs}$ were more frequently detected in $\mathrm{HBV}^{-} \mathrm{HCCs}$ than in $\mathrm{HCV}$-related HCCs, although a validation study using a large independent cohort is required (18). A recent study supported the role of $\mathrm{HBx}$ in the activation of HepG2 cell EpCAM expression accompanied by enhanced cell migration and sphere formation (133). Continued expression of HCV using a subgenomic replicon system was shown to induce stem cell-like properties, including the activation of CD133, AFP, CK19, and c-Myc (134). Moreover, TLR4 was induced in HCV NS5A transgenic mice following alcohol exposure, and the resulting HCC showed activation of stem cell signatures including CD133 and Nanog (106). As liver CSCs are considered resistant to cellular stresses, it should be clarified whether HBV or HCV infection directly induces stemness through interaction with signaling pathways, or whether the results reflect enrichment of stress-resistant CSCs in certain conditions.

The target cell population of malignant transformation is generally controversial in human cancer, but accumulating evidence suggests that cancer heterogeneity may derive from different cells of origin as well as diverse genetic mutations (135). Recent studies have indicated the spontaneous conversion of non-stem cells to stem-like cells in normal breast epithelial cells and suggested that the biological state of normal cells of origin before transformation may be a determinant of the behavior of their descendants following transformation (136). Similarly, a recent study suggested the unexpected plasticity of normal mature hepatocytes to dedifferentiate into progenitor cells in rodent 
(137), but the relation between the biological state of cells of origin in liver lineages and the descendant liver cancer phenotypes remain to be clarified (138-141). Interestingly, a recent study demonstrated that ICC originates from hepatocytes when signaling of Notch and Akt is activated in mice, suggesting a role for hepatobiliary lineage commitment program deregulation in hepatocarcinogenesis (Figure 2 and ref. 142). In rodents, significant studies have demonstrated that HCC may originate from oval cells as well as hepatocytes (see also a recent review article summarizing important earlier works of experimental chemical hepatocarcinogenesis models; ref. 141). However, the cellular origin of human HCC, ICC, and HCC-CCA remains elusive.

Stem cell niches have been identified and characterized in many tissues, including the germline, bone marrow, intestine, muscle, skin, hair follicle, mammary gland, and nervous system (143). However, the niche cells that control the proliferation and selfrenewal of liver CSCs as well as normal hepatic stem/progenitors have not yet been clarified.

Current knowledge of CSCs is influenced largely by the biology of normal stem cells, in terms of activated markers and signaling pathways. Therefore, elimination of liver CSCs using these markers and signaling pathways may reduce normal hepatic stem/ progenitor cells in CLDs, which may inhibit hepatic regeneration leading to hepatic failure. Thus far, it is unclear whether liver CSCs can be effectively eliminated without affecting normal hepatic stem/progenitor cells. In leukemia, PI3K and downstream mTOR kinase composed of key complexes mTORC1 and mTORC2 are frequently activated in CSCs. Recent studies demonstrated a nonredundant requirement of $\mathrm{mTORC} 1$ for both hematopoiesis and PTEN loss-induced leukemogenesis in mice $(144,145)$. Interestingly, mTORC2 signaling was also required for leukemogenesis but had little effect on normal hematopoietic stem cell function in PTEN-null adult mice, suggesting that mTORC2 is a potential target for the eradication of leukemia CSCs without affecting normal adult hematopoiesis (145).

According to the conventional CSC model, only CSCs are highly tumorigenic and metastatic and can divide asymmetrically to generate non-CSCs, and the frequency of CSCs is maintained at a low level. Therefore, eradication of CSCs alone is considered sufficient for tumor regression. However, recent evidence suggests that nonCSCs de-differentiate to generate CSCs in breast cancer $(136,146$, 147). Hepatocytes may have similar features to stem cells in terms of self-renewal, biliary differentiation, and unlimited cell proliferation under certain conditions $(27,81,148,149)$. It is plausible that transformed marker-negative cancer cells de-differentiate to acquire features of liver CSCs (Figure 2).

In leukemia, the frequency of CSCs is not always maintained at low levels, and clonal evolution by genetic changes may determine the nature and frequency of CSCs $(11,150)$. Similarly, the frequency of $\mathrm{CD} 133^{+}$or $\mathrm{EpCAM}^{+} \mathrm{HCC}$ increases as tumors advance $(18,128)$, and the clonal evolution model in collaboration with the CSC model could explain the emergence of certain liver CSCs at later stages. Should this be the case, it is unclear whether liver CSCs exist in all liver cancers at every stage. Moreover, given the diversity of HCC genetic traits revealed by whole genome sequencing, it is reasonable to speculate that no common liver CSCs expressing certain stem cell markers exist in all liver cancers. Rather, liver CSCs are likely to be distinct and different for each cancer according to genetic traits and activated signaling pathways. This warrants further studies to provide better diagnostic and treatment strategies for liver cancer patients.

\section{Acknowledgments}

We apologize to those investigators whose original works were not cited due to space limitations. T. Yamashita is supported by a grant from the Ministry of Education, Culture, Sports, Science and Technology and the National Cancer Center Research and Development Fund of Japan. X.W. Wang is supported by the Intramural Research Program of the Center for Cancer Research, US National Cancer Institute (grant Z01 BC010876).

Address correspondence to: Taro Yamashita, Department of General Medicine, Kanazawa University Hospital, Kanazawa, Ishikawa 920-8641, Japan. Phone: 81.76.265.2042; Fax: 81.76.234.4250; E-mail: taroy@m-kanazawa.jp. Or to: Xin Wei Wang, Laboratory of Human Carcinogenesis, Center for Cancer Research, National Cancer Institute, Bethesda, Maryland 20892-4255, USA. Phone: 301.496.2099; Fax: 301.496.0497; E-mail:xw3u@nih.gov.
1. Fialkow PJ. Clonal origin of human tumors. Biochim Biophys Acta. 1976;458(3):283-321.

2. Fidler IJ, Kripke ML. Metastasis results from preexisting variant cells within a malignant tumor. Science. 1977;197(4306):893-895.

3. Nowell PC. The clonal evolution of tumor cell populations. Science. 1976;194(4260):23-28.

4. Baylin SB, Jones PA. A decade of exploring the cancer epigenome - biological and translational implications. Nat Rev Cancer. 2011;11(10):726-734.

5. Mueller MM, Fusenig NE. Friends or foes - bipolar effects of the tumour stroma in cancer. Nat Rev Cancer. 2004;4(11):839-849.

6. Jordan CT, Guzman ML, Noble M. Cancer stem cells. N Engl J Med. 2006;355(12):1253-1261.

7. Cohnheim J. Congenitales, quergestreiftes Muskelsarkom der Nieren. Path Anat Physiol Klin Med. 1875; 65:64-69.

8. Wicha MS, Liu S, Dontu G. Cancer stem cells: an old idea - a paradigm shift. Cancer Res. 2006; 66(4):1883-1890

9. Bonnet D, Dick JE. Human acute myeloid leukemia is organized as a hierarchy that originates from a primitive hematopoietic cell. Nat Med. 1997; 3(7):730-737.

10. Lapidot $\mathrm{T}$, et al. A cell initiating human acute myeloid leukaemia after transplantation into SCID mice. Nature. 1994;367(6464):645-648.

11. Magee JA, Piskounova E, Morrison SJ. Cancer stem cells: impact, heterogeneity, and uncertainty. Cancer Cell. 2012;21(3):283-296.

12. Jemal A, Bray F, Center MM, Ferlay J, Ward E, Forman D. Global cancer statistics. CA Cancer J Clin. 2011;61(2):69-90.

13. de Jong MC, et al. Intrahepatic cholangiocarcinoma: an international multi-institutional analysis of prognostic factors and lymph node assessment. J Clin Oncol. 2011;29(23):3140-3145.

14. Zhang F, et al. Combined hepatocellular cholangiocarcinoma originating from hepatic progenitor cells: immunohistochemical and double-fluorescence immunostaining evidence. Histopathology. 2008;52(2):224-232.

15. Cardinale V, Wang Y, Carpino G, Reid LM, Gaudio E, Alvaro D. Mucin-producing cholangiocarcinoma might derive from biliary tree stem/progenitor cells located in peribiliary glands. Hepatology. 2012;55(6):2041-2042.

16. Komuta M, et al. Clinicopathological study on cholangiolocellular carcinoma suggesting hepatic progenitor cell origin. Hepatology. 2008;47(5):1544-1556.

17. Ma S, et al. Identification and characterization of tumorigenic liver cancer stem/progenitor cells. Gastroenterology. 2007;132(7):2542-2556.

18. Yamashita T, et al. EpCAM-positive hepatocellular carcinoma cells are tumor-initiating cells with stem/ progenitor cell features. Gastroenterology. 2009; 136(3):1012-1024.

19. Haraguchi $\mathrm{N}$, et al. CD13 is a therapeutic target in human liver cancer stem cells. J Clin Invest. 2010; 120(9):3326-3339.

20. Yang ZF, et al. Significance of CD90+ cancer stem cells in human liver cancer. Cancer Cell. 2008; 13(2):153-166.

21. Lee TK, Castilho A, Cheung VC, Tang KH, Ma S, $\mathrm{Ng}$ IO. CD24(+) liver tumor-initiating cells drive self-renewal and tumor initiation through STAT3mediated NANOG regulation. Cell Stem Cell. 2011; 9(1):50-63.

22. Ma S, et al. Aldehyde dehydrogenase discriminates the CD133 liver cancer stem cell populations. Mol Cancer Res. 2008;6(7):1146-1153.

23. Yang W, et al. OV6(+) tumor-initiating cells contribute to tumor progression and invasion in human hepatocellular carcinoma. J Hepatol. 2012; 57(3):613-620.

24. Chiba T, et al. Side population purified from hepatocellular carcinoma cells harbors cancer stem cell- 
like properties. Hepatology. 2006;44(1):240-251.

25. Thorgeirsson SS, Grisham JW. Molecular pathogenesis of human hepatocellular carcinoma. Nat Genet. 2002;31(4):339-346.

26. Roskams T. Liver stem cells and their implication in hepatocellular and cholangiocarcinoma. Oncogene. 2006;25(27):3818-3822.

27. Oertel M, Shafritz DA. Stem cells, cell transplantation and liver repopulation. Biochim Biophys Acta. 2008;1782(2):61-74.

28. Nguyen H, Mudryj M, Guadalupe M, Dandekar S. Hepatitis $\mathrm{C}$ virus core protein expression leads to biphasic regulation of the p $21 \mathrm{cdk}$ inhibitor and modulation of hepatocyte cell cycle. Virology. 2003;312(1):245-253

29. Marshall A, et al. Relation between hepatocyte G1 arrest, impaired hepatic regeneration, and fibrosis in chronic hepatitis $\mathrm{C}$ virus infection. Gastroenterology. 2005;128(1):33-42.

30. Clouston AD, Powell EE, Walsh MJ, Richardson MM, Demetris AJ, Jonsson JR. Fibrosis correlates with a ductular reaction in hepatitis C: roles of impaired replication, progenitor cells and steatosis. Hepatology. 2005;41(4):809-818.

31. Saxena R, Theise N. Canals of Hering: recent insights and current knowledge. Semin Liver Dis. 2004; 24(1):43-48.

32. Scadden DT. The stem-cell niche as an entity of action. Nature. 2006;441(7097):1075-1079.

33. Ziol M, et al. Intermediate hepatobiliary cells predict an increased risk of hepatocarcinogenesis in patients with hepatitis $C$ virus-related cirrhosis. Gastroenterology. 2010;139(1):335-343.

34. Feitelson MA, Lee J. Hepatitis B virus integration, fragile sites, and hepatocarcinogenesis. Cancer Lett. 2007;252(2):157-170.

35. Wang XW, Forrester K, Yeh H, Feitelson MA, Gu JR, Harris CC. Hepatitis B virus X protein inhibits p53 sequence-specific DNA binding, transcriptional activity, and association with transcription factor ERCC3. Proc Natl Acad Sci U S A. 1994;91(6):2230-2234.

36. Okuda $M$, et al. Mitochondrial injury, oxidative stress, and antioxidant gene expression are induced by hepatitis C virus core protein. Gastroenterology. 2002;122(2):366-375

37. Farazi PA, DePinho RA. Hepatocellular carcinoma pathogenesis: from genes to environment. Nat Rev Cancer. 2006;6(9):674-687.

38. Hussain SP, Schwank J, Staib F, Wang XW, Harris CC. TP53 mutations and hepatocellular carcinoma: insights into the etiology and pathogenesis of liver cancer. Oncogene. 2007;26(15):2166-2176.

39. Budhu A, et al. Prediction of venous metastases, recurrence, and prognosis in hepatocellular carcinoma based on a unique immune response signature of the liver microenvironment. Cancer Cell. 2006;10(2):99-111.

40. Bataller R, Brenner DA. Liver fibrosis. J Clin Invest. 2005;115(2):209-218

41. Gressner AM. Cytokines and cellular crosstalk involved in the activation of fat-storing cells. J Hepatol. 1995;22(2 suppl):28-36.

42. Thompson MD, Monga SP. WNT/beta-catenin signaling in liver health and disease. Hepatology. 2007;45(5):1298-1305.

43. Sato T, El-Assal ON, Ono T, Yamanoi A, Dhar DK, Nagasue N. Sinusoidal endothelial cell proliferation and expression of angiopoietin/Tie family in regenerating rat liver. J Hepatol. 2001;34(5):690-698.

44. Shimizu H, et al. Vascular endothelial growth factor secreted by replicating hepatocytes induces sinusoidal endothelial cell proliferation during regeneration after partial hepatectomy in rats. J Hepatol. 2001;34(5):683-689.

45. Jung J, Zheng M, Goldfarb M, Zaret KS. Initiation of mammalian liver development from endoderm by fibroblast growth factors. Science. 1999; 284(5422):1998-2003.
46. Rossi JM, Dunn NR, Hogan BL, Zaret KS. Distinct mesodermal signals, including BMPs from the septum transversum mesenchyme, are required in combination for hepatogenesis from the endoderm. Genes Dev. 2001;15(15):1998-2009.

47. Ober EA, Verkade H, Field HA, Stainier DY. Mesodermal Wnt2b signalling positively regulates liver specification. Nature. 2006;442(7103):688-691.

48. Decaens T, et al. Stabilization of beta-catenin affects mouse embryonic liver growth and hepatoblast fate. Hepatology. 2008;47(1):247-258.

49. Kinoshita T, Miyajima A. Cytokine regulation of liver development. Biochim Biophys Acta. 2002; 1592(3):303-312.

50. Lemaigre F, Zaret KS. Liver development update: new embryo models, cell lineage control, and morphogenesis. Curr Opin Genet Dev. 2004;14(5):582-590.

51. Monga SP, Monga HK, Tan X, Mule K, Pediaditakis $\mathrm{P}$, Michalopoulos GK. Beta-catenin antisense studies in embryonic liver cultures: role in proliferation, apoptosis, and lineage specification. Gastroenterology. 2003;124(1):202-216.

52. Ji J, Wang XW. Clinical implications of cancer stem cell biology in hepatocellular carcinoma. Semin Oncol. 2012;39(4):461-472.

53. Gauglhofer C, et al. Up-regulation of the fibroblast growth factor 8 subfamily in human hepatocellular carcinoma for cell survival and neoangiogenesis. Hepatology. 2011;53(3):854-864.

54. Whittaker S, Marais R, Zhu AX. The role of signaling pathways in the development and treatment of hepatocellular carcinoma. Oncogene. 2010; 29(36):4989-5005.

55. Merle P, et al. Functional consequences of frizzled-7 receptor overexpression in human hepatocellular carcinoma. Gastroenterology. 2004;127(4):1110-1122.

56. Yamashita T, Budhu A, Forgues M, Wang XW. Activation of hepatic stem cell marker EpCAM by Wnt-beta-catenin signaling in hepatocellular carcinoma. Cancer Res. 2007;67(22):10831-10839.

57. Yamashita T, et al. EpCAM and alpha-fetoprotein expression defines novel prognostic subtypes of hepatocellular carcinoma. Cancer Res. 2008; 68(5):1451-1461.

58 . Yang W, et al. Wnt/beta-catenin signaling contributes to activation of normal and tumorigenic liver progenitor cells. Cancer Res. 2008;68(11):4287-4295.

59. Apte U, Thompson MD, Cui S, Liu B, Cieply B, Monga SP. Wnt/beta-catenin signaling mediates oval cell response in rodents. Hepatology. 2008; 47(1):288-295.

60 . Ji J, et al. Identification of microRNA-181 by genome-wide screening as a critical player in EpCAM-positive hepatic cancer stem cells. Hepatology. 2009;50(2):472-480.

61. Guichard C, et al. Integrated analysis of somatic mutations and focal copy-number changes identifies key genes and pathways in hepatocellular carcinoma. Nat Genet. 2012;44(6):694-698.

62. Fujimoto A, et al. Whole-genome sequencing of liver cancers identifies etiological influences on mutation patterns and recurrent mutations in chromatin regulators. Nat Genet. 2012;44(7):760-764.

63. Li M, et al. Inactivating mutations of the chromatin remodeling gene ARID2 in hepatocellular carcinoma. Nat Genet. 2011;43(9):828-829.

64 . Hoshida $Y$, et al. Integrative transcriptome analysis reveals common molecular subclasses of human hepatocellular carcinoma. Cancer Res. 2009; 69(18):7385-7392.

65. Coulouarn C, et al. Combined hepatocellular-cholangiocarcinomas exhibit progenitor features and activation of Wnt and TGFbeta signaling pathways. Carcinogenesis. 2012;33(9):1791-1796.

66. Zhang L, et al. BMP4 administration induces differentiation of CD133+ hepatic cancer stem cells, blocking their contributions to hepatocellular carcinoma. Cancer Res. 2012;72(16):4276-4285.
67. Yamashita $\mathrm{T}$, et al. Oncostatin $\mathrm{m}$ renders epithelial cell adhesion molecule-positive liver cancer stem cells sensitive to 5 -Fluorouracil by inducing hepatocytic differentiation. Cancer Res. 2010; 70(11):4687-4697.

68. Clotman F, et al. Control of liver cell fate decision by a gradient of TGF beta signaling modulated by Onecut transcription factors. Genes Dev. 2005; 19(16):1849-1854.

69. Thenappan A, et al. Role of transforming growth factor beta signaling and expansion of progenitor cells in regenerating liver. Hepatology. 2010;51(4):1373-1382.

70. Mishra L, et al. Liver stem cells and hepatocellular carcinoma. Hepatology. 2009;49(1):318-329.

71. Morris SM, Baek JY, Koszarek A, Kanngurn S, Knoblaugh SE, Grady WM. Transforming growth factor-beta signaling promotes hepatocarcinogenesis induced by p53 loss. Hepatology. 2012;55(1):121-131.

72. Wu K, et al. Hepatic TGF-beta gives rise to tumor-initiating cells and promotes liver cancer development. Hepatology. 2012;56(6):2255-2267.

73. You H, Ding W, Rountree CB. Epigenetic regulation of cancer stem cell marker CD133 by transforming growth factor-beta. Hepatology. 2010; 51(5):1635-1644.

74. Budhu A, Wang XW. Transforming the microenvironment: a trick of the metastatic cancer cell. Cancer Cell. 2012;22(3):279-280.

75. Yang $P$, et al. TGF-beta-miR-34a-CCL22 signalinginduced Treg cell recruitment promotes venous metastases of HBV-positive hepatocellular carcinoma. Cancer Cell. 2012;22(3):291-303.

76. Michalopoulos GK. Liver regeneration. J Cell Physiol. 2007;213(2):286-300.

77. Tang Y, et al. Progenitor/stem cells give rise to liver cancer due to aberrant TGF-beta and IL-6 signaling. Proc Natl Acad Sci U S A. 2008;105(7):2445-2450.

78. Viatour $P$, et al. Notch signaling inhibits hepatocellular carcinoma following inactivation of the RB pathway. J Exp Med. 2011;208(10):1963-1976.

79. Villanueva A, et al. Notch signaling is activated in human hepatocellular carcinoma and induces tumor formation in mice. Gastroenterology. 2012; 143(6):1660-1669.

80. Ishikawa T, et al. Hepatocyte growth factor/c-met signaling is required for stem-cell-mediated liver regeneration in mice. Hepatology. 2012;55(4):1215-1226.

81. Limaye PB, Bowen WC, Orr AV, Luo J, Tseng GC, Michalopoulos GK. Mechanisms of hepatocyte growth factor-mediated and epidermal growth factor-mediated signaling in transdifferentiation of rat hepatocytes to biliary epithelium. Hepatology. 2008;47(5):1702-1713.

82. Ding W, et al. Epithelial-to-mesenchymal transition of murine liver tumor cells promotes invasion. Hepatology. 2010;52(3):945-953.

83. Schmelzer E, et al. Human hepatic stem cells from fetal and postnatal donors. J Exp Med. 2007; 204(8):1973-1987.

84. Niki T, et al. Class VI intermediate filament protein nestin is induced during activation of rat hepatic stellate cells. Hepatology. 1999;29(2):520-527.

85. Roskams T, Cassiman D, De Vos R, Libbrecht L. Neuroregulation of the neuroendocrine compartment of the liver. Anat Rec A Discov Mol Cell Evol Biol. 2004;280(1):910-923.

86. Crosby HA, Kelly DA, Strain AJ. Human hepatic stem-like cells isolated using c-kit or CD34 can differentiate into biliary epithelium. Gastroenterology. 2001;120(2):534-544.

87. Suzuki A, et al. Flow cytometric isolation and clonal identification of self-renewing bipotent hepatic progenitor cells in adult mouse liver. Hepatology. 2008;48(6):1964-1978.

88. Rountree CB, Barsky L, Ge S, Zhu J, Senadheera S, Crooks GM. A CD133-expressing murine liver oval cell population with bilineage potential. Stem Cells. 2007;25(10):2419-2429. 
89. Weiss TS, et al. Hepatic progenitor cells from adult human livers for cell transplantation. Gut. 2008;57(8):1129-1138.

90. Nitou M, Sugiyama Y, Ishikawa K, Shiojiri N. Purification of fetal mouse hepatoblasts by magnetic beads coated with monoclonal anti-e-cadherin antibodies and their in vitro culture. Exp Cell Res. 2002; 279(2):330-343.

91. Oertel M, Menthena A, Chen YQ, Teisner B, Jensen $\mathrm{CH}$, Shafritz DA. Purification of fetal liver stem/ progenitor cells containing all the repopulation potential for normal adult rat liver. Gastroenterology. 2008;134(3):823-832.

92. Tanimizu N, Nishikawa M, Saito H, Tsujimura $\mathrm{T}$, Miyajima A. Isolation of hepatoblasts based on the expression of Dlk/Pref-1. J Cell Sci. 2003; 116(pt 9):1775-1786.

93. Roskams T, De Vos R, Van Eyken P, Myazaki H, Van Damme B, Desmet V. Hepatic OV-6 expression in human liver disease and rat experiments: evidence for hepatic progenitor cells in man.J Hepatol. 1998; 29(3):455-463.

94. Van Den Heuvel MC, et al. Expression of anti-OV6 antibody and anti-N-CAM antibody along the biliary line of normal and diseased human livers. Hepa tology. 2001;33(6):1387-1393

95. Yovchev MI, Grozdanov PN, Zhou H, Racherla H, Guha C, Dabeva MD. Identification of adult hepatic progenitor cells capable of repopulating injured rat liver. Hepatology. 2008;47(2):636-647.

96. Qiu Q, Hernandez JC, Dean AM, Rao PH, Darlington GJ. CD24-positive cells from normal adult mouse liver are hepatocyte progenitor cells. Stem Cells Dev. 2011;20(12):2177-2188.

97. Okada K, et al. Prospective isolation and characterization of bipotent progenitor cells in early mouse liver development. Stem Cells Dev. 2012;21(7):1124-1133.

98. Xu X, et al. DLK1 as a potential target against cancer stem/progenitor cells of hepatocellular carcinoma. Mol Cancer Ther. 2012;11(3):629-638.

99. Maetzel D, et al. Nuclear signalling by tumourassociated antigen EpCAM. Nat Cell Biol. 2009; 11(2):162-171.

100. Huang HP, et al. Epithelial cell adhesion molecule (EpCAM) complex proteins promote transcription factor-mediated pluripotency reprogramming. J Biol Chem. 2011;286(38):33520-33532.

101. Tang $\mathrm{KH}$, et al. CD133(+) liver tumor-initiating cells promote tumor angiogenesis, growth, and self-renewal through neurotensin/interleukin-8/ CXCL1 signaling. Hepatology. 2012;55(3):807-820.

102.Ishimoto $\mathrm{T}$, et al. CD44 variant regulates redox status in cancer cells by stabilizing the $\mathrm{xCT}$ subunit of system $\mathrm{xc}(-)$ and thereby promotes tumor growth. Cancer Cell. 2011;19(3):387-400.

103.Takahashi K, et al. Induction of pluripotent stem cells from adult human fibroblasts by defined factors. Cell. 2007;131(5):861-872.

104. Takahashi K, Yamanaka S. Induction of pluripotent stem cells from mouse embryonic and adult fibroblast cultures by defined factors. Cell. 2006;126(4):663-676.

105. Chow EK, Fan LL, Chen X, Bishop JM. Oncogenespecific formation of chemoresistant murine hepatic cancer stem cells. Hepatology. 2012; 56(4):1331-1341.

106. Machida K, et al. Toll-like receptor 4 mediates synergism between alcohol and HCV in hepatic oncogenesis involving stem cell marker Nanog. Proc Natl Acad Sci U S A. 2009;106(5):1548-1553

107. Huang P, et al. Role of Sox 2 and Oct 4 in predicting survival of hepatocellular carcinoma patients after hepatectomy. Clin Biochem. 2011;44(8-9):582-589.
108. Yin X, et al. Coexpression of stemness factors oct 4 and nanog predict liver resection. Ann Surg Oncol. 2012;19(9):2877-2887.

109. Woo HG, et al. Association of TP53 mutations with stem cell-like gene expression and survival of patients with hepatocellular carcinoma. Gastroenterology. 2011;140(3):1063-1070.

110 .Hong H, et al. Suppression of induced pluripotent stem cell generation by the p53-p21 pathway. Nature. 2009;460(7259):1132-1135.

111. Marion RM, et al. A p53-mediated DNA damage response limits reprogramming to ensure iPS cell genomic integrity. Nature. 2009;460(7259):1149-1153.

112. Utikal $\mathrm{J}$, et al. Immortalization eliminates a roadblock during cellular reprogramming into iPS cells. Nature. 2009;460(7259):1145-1148.

113.Jelnes P, et al. Remarkable heterogeneity displayed by oval cells in rat and mouse models of stem cell-mediated liver regeneration. Hepatology. 2007; 45(6):1462-1470

114. Oertel M, Menthena A, Chen YQ, Shafritz DA. Comparison of hepatic properties and transplantation of Thy-1(+) and Thy-1(-) cells isolated from embryonic day 14 rat fetal liver. Hepatology. 2007; 46(4):1236-1245

115.Dan YY, et al. Isolation of multipotent progenitor cells from human fetal liver capable of differentiating into liver and mesenchymal lineages. Proc Natl Acad SciU S A. 2006;103(26):9912-9917.

116.Yamashita T, et al. Discrete nature of $\operatorname{EpCAM}(+)$ and $\mathrm{CD} 90(+)$ cancer stem cells in human hepatocellular carcinoma. Hepatology. 2013;57(4):1484-1497.

117. Hoshida Y, et al. Gene expression in fixed tissues and outcome in hepatocellular carcinoma. $N$ EnglJ Med. 2008;359(19):1995-2004.

118.Ji J, et al. MicroRNA expression, survival, and response to interferon in liver cancer. $N$ Engl J Med. 2009;361(15):1437-1447

119. Cairo S, et al. Stem cell-like micro-RNA signature driven by Myc in aggressive liver cancer. Proc Natl Acad Sci U S A. 2010;107(47):20471-20476.

120.Lee JS, et al. A novel prognostic subtype of human hepatocellular carcinoma derived from hepatic progenitor cells. Nat Med. 2006;12(4):410-416.

121. Kim H, et al. Human hepatocellular carcinomas with "Stemness"-related marker expression: keratin 19 expression and a poor prognosis. Hepatology. 2011;54(5):1707-1717.

122.Mima K, et al. CD44s regulates the TGF-beta-mediated mesenchymal phenotype and is associated with poor prognosis in patients with hepatocellular carcinoma. Cancer Res. 2012;72(13):3414-3423.

123.Yang XR, et al. High expression levels of putative hepatic stem/progenitor cell biomarkers related to tumour angiogenesis and poor prognosis of hepatocellular carcinoma. Gut. 2010;59(7):953-962.

124. Oishi N, et al. Transcriptomic profiling reveals hepatic stem-like gene signatures and interplay of miR-200c and epithelial-mesenchymal transition in intrahepatic cholangiocarcinoma. Hepatology. 2012; 56(5):1792-1803

125.Yang ZF, et al. Identification of local and circulating cancer stem cells in human liver cancer. Hepatology. 2008;47(3):919-928.

126. Kim MY, et al. Tumor self-seeding by circulating cancer cells. Cell. 2009;139(7):1315-1326.

127. Sun YF, et al. Circulating stem cell-like epithelial cell adhesion molecule-positive tumor cells indicate poor prognosis of hepatocellular carcinoma after curative resection. Hepatology. 2013;57(4):1458-1468.

128. Ma S, et al. miR-130b Promotes CD133(+) liver tumor-initiating cell growth and self-renewal via tumor protein 53-induced nuclear protein 1. Cell Stem Cell. 2010;7(6):694-707.

129. de Lope CR, Tremosini S, Forner A, Reig M, Bruix J. Management of HCC. J Hepatol. 2012; 56(suppl 1):S75-S87.

130.Ma S, Lee TK, Zheng BJ, Chan KW, Guan XY. CD133+ HCC cancer stem cells confer chemoresistance by preferential expression of the Akt/PKB survival pathway. Oncogene. 2008;27(12):1749-1758.

131.Lee TK, Castilho A, Cheung VC, Tang KH, Ma S, $\mathrm{Ng}$ IO. Lupeol targets liver tumor-initiating cells through phosphatase and tensin homolog modulation. Hepatology. 2011;53(1):160-170.

132. Lazaro CA, et al. Hepatitis $C$ virus replication in transfected and serum-infected cultured human fetal hepatocytes. Am J Pathol. 2007;170(2):478-489.

133.Arzumanyan A, Friedman T, Ng IO, Clayton MM, Lian Z, Feitelson MA. Does the hepatitis B antigen HBx promote the appearance of liver cancer stem cells? Cancer Res. 2011;71(10):3701-3708.

134.Ali N, et al. Hepatitis C virus-induced cancer stem cell-like signatures in cell culture and murine tumor xenografts. J Virol. 2011;85(23):12292-12303.

135.Visvader JE. Cells of origin in cancer. Nature. 2011; 469(7330):314-322

136. Chaffer CL, et al. Normal and neoplastic nonstem cells can spontaneously convert to a stem-like state. Proc Natl Acad Sci U S A. 2011;108(19):7950-7955.

137. Chen Y, Wong PP, Sjeklocha L, Steer CJ, Sahin MB. Mature hepatocytes exhibit unexpected plasticity by direct dedifferentiation into liver progenitor cells in culture. Hepatology. 2012;55(2):563-574.

138. Chiba T, et al. Enhanced self-renewal capability in hepatic stem/progenitor cells drives cancer initiation. Gastroenterology. 2007;133(3):937-950

139. Sell S. Cellular origin of hepatocellular carcinomas. Semin Cell Dev Biol. 2002;13(6):419-424.

140.Zender L, et al. Identification and validation of oncogenes in liver cancer using an integrative oncogenomic approach. Cell. 2006;125(7):1253-1267.

141.Sell S, Leffert HL. Liver cancer stem cells. J Clin Oncol. 2008;26(17):2800-2805.

142.Fan B, et al. Cholangiocarcinomas can originate from hepatocytes in mice. J Clin Invest. 2012; 122(8):2911-2915.

143. Wagers AJ. The stem cell niche in regenerative medicine. Cell Stem Cell. 2012;10(4):362-369.

144. Kalaitzidis D, et al. mTOR complex 1 plays critical roles in hematopoiesis and Pten-loss-evoked leukemogenesis. Cell Stem Cell. 2012;11(3):429-439.

145. Magee JA, Ikenoue T, Nakada D, Lee JY, Guan KL, Morrison SJ. Temporal changes in PTEN and mTORC2 regulation of hematopoietic stem cell self-renewal and leukemia suppression. Cell Stem Cell. 2012;11(3):415-428.

146.Gupta PB, et al. Stochastic state transitions give rise to phenotypic equilibrium in populations of cancer cells. Cell. 2011;146(4):633-644.

147. Mani SA, et al. The epithelial-mesenchymal transition generates cells with properties of stem cells. Cell. 2008;133(4):704-715.

148. Michalopoulos GK, Barua L, Bowen WC. Transdifferentiation of rat hepatocytes into biliary cells after bile duct ligation and toxic biliary injury. Hepatology. 2005;41(3):535-544.

149. Overturf K, al-Dhalimy M, Ou CN, Finegold M, Grompe M. Serial transplantation reveals the stem-cell-like regenerative potential of adult mouse hepatocytes. Am J Pathol. 1997;151(5):1273-1280.

150. Notta F, et al. Evolution of human BCR-ABL1 lymphoblastic leukaemia-initiating cells. Nature. 2011;469(7330):362-367. 